\title{
Analysis of Mutations in AARS2 in a Series of CSF1R-Negative Patients With Adult-Onset Leukoencephalopathy With Axonal Spheroids and Pigmented Glia
}

David S. Lynch, MRCPI; Wei Jia Zhang, MRCP; Rahul Lakshmanan, FRANZCR; Justin A. Kinsella, PhD; Güneş Altıokka Uzun, MD; Merih Karbay, MD; Zeynep Tüfekçioğlu, MD; Haşmet Hanağası, MD; Georgina Burke, PhD; Nicola Foulds, FRCP; Simon R. Hammans, FRCP; Anupam Bhattacharjee, PhD; Heather Wilson, FRCP; Matthew Adams, FRCR; Mark Walker, FRCPath; James A. R. Nicoll, FRCPath; Jeremy Chataway, FRCP; Nick Fox, FRCP; Indran Davagnanam, FRCR;

Rahul Phadke, FRCPath; Henry Houlden, PhD

IMPORTANCE Adult-onset leukoencephalopathy with axonal spheroids and pigmented glia (ALSP) is a frequent cause of adult-onset leukodystrophy known to be caused by autosomal dominant mutations in the CSF1R (colony-stimulating factor 1) gene. The discovery that CSF1R mutations cause ALSP led to more accurate prognosis and genetic counseling for these patients in addition to increased interest in microglia as a target in neurodegeneration. However, it has been known since the discovery of the CSF1R gene that there are patients with typical clinical and radiologic evidence of ALSP who do not carry pathogenic CSF1R mutations. These patients include those in whom the pathognomonic features of axonal spheroids and pigmented microglia have been found. Achieving a genetic diagnosis in these patients is important to our understanding of this disorder.

OBJECTIVE To genetically characterize a group of patients with typical features of ALSP who do not carry CSF1R mutations.

DESIGN, SETTINGS, AND PARTICIPANTS In this case series study, 5 patients from 4 families were identified with clinical, radiologic, or pathologic features of ALSP in whom CSF1R mutations had been excluded previously by sequencing. Data were collected between May 2014 and September 2015 and analyzed between September 2015 and February 2016.

MAIN OUTCOMES AND MEASURES Focused exome sequencing was used to identify candidate variants. Family studies, long-range polymerase chain reaction with cloning, and complementary DNA sequencing were used to confirm pathogenicity.

RESULTS Of these 5 patients, 4 were men (80\%); mean age at onset of ALSP was 29 years (range, 15-44 years). Biallelic mutations in the alanyl-transfer (t)RNA synthetase 2 (AARS2) gene were found in all 5 patients. Frameshifting and splice site mutations were common, found in 4 of 5 patients, and sequencing of complementary DNA from affected patients confirmed that the variants were loss of function. All patients presented in adulthood with prominent cognitive, neuropsychiatric, and upper motor neuron signs. Magnetic resonance imaging in all patients demonstrated a symmetric leukoencephalopathy with punctate regions of restricted diffusion, typical of ALSP. In 1 patient, brain biopsy demonstrated axonal spheroids and pigmented microglia, which are the pathognomonic signs of ALSP.

CONCLUSIONS AND RELEVANCE This work indicates that mutations in the tRNA synthetase AARS2 gene cause a recessive form of ALSP. The CSF1R and AARS2 proteins have different cellular functions but overlap in a final common pathway of neurodegeneration. This work points to novel targets for research and will lead to improved diagnostic rates in patients with adult-onset leukoencephalopathy.

JAMA Neurol. 2016;73(12):1433-1439. doi:10.1001/jamaneurol.2016.2229

Published online October 17, 2016.

\author{
Author Affiliations: Author \\ affiliations are listed at the end of this \\ article. \\ Corresponding Author: David S. \\ Lynch, MRCPI, Department of \\ Molecular Neuroscience, University \\ College London Institute of \\ Neurology, Queen Square, \\ London WC1N 3BG, England \\ (david.lynch.13@ucl.ac.uk).
}


A dult-onset genetic leukoencephalopathies comprise a diverse group of degenerative white matter disorders with an ever-increasing number of associated genes. ${ }^{1}$ Most patients present with a variable phenotype of cognitive decline, neuropsychiatric disturbance, and pyramidal and extrapyramidal signs. The often-overlapping symptoms and frequently similar magnetic resonance imaging (MRI) patterns mean that, even with systematic testing, one-third of patients do not receive a genetic diagnosis. ${ }^{2}$ In addition, the cost of comprehensive metabolic and genetic screening may be prohibitive in many health care settings. Despite these factors, definitive genetic diagnosis is the only way that patients can be given appropriate genetic counseling or a relatively accurate prognosis.

Adult-onset leukoencephalopathy with axonal spheroids and pigmented glia (ALSP) is the most frequent adult-onset leukodystrophy and is caused by autosomal dominant mutations in the CSF1R (colony-stimulating factor 1) gene (OMIM: 164770). ${ }^{3}$ The clinical and pathologic features of ALSP are well defined $^{4-6}$; however, several apparently typical cases have been shown ${ }^{7}$ not to possess CSF1R mutations.

Alanyl-transfer (t)RNA synthetase 2 (AARS2) is a gene (OMIM: 612035) encoding the tRNA synthetase responsible for loading alanine (ala) onto tRNA-ala during mitochondrial translation. In 2011, the first association of AARS2 mutations and human disease was published, ${ }^{8}$ when 3 patients with fatal infantile cardiomyopathy were described with autosomal recessive mutations in the gene. Later, Dallabona et $\mathrm{al}^{9}$ described 6 patients with ovarioleukodystrophy who were found to have AARS2 mutations by whole-exome sequencing. The MRI appearance was nonspecific and consisted of signal abnormality in the frontal, parietal, and periventricular white matter with involvement of the corpus callosum and patchy areas of restricted diffusion on diffusion-weighted imaging. No brain tissue was available for pathologic examination in any AARS2 cases.

In this study, we describe 5 patients with an adult-onset phenotype typical of ALSP who were found to carry compound heterozygous or homozygous mutations in AARS2. Of these patients, 4 were men (80\%); mean age at onset of ALSP was 29 years (range, $15-44$ years). In addition, we describe, for what we believe to be the first time, the central nervous system pathologic features of AARS2-related leukoencephalopathy in which frequent axonal spheroids and pigmented microglia were seen. We provide an explanation for several patients with CSF1R-negative ALSP, particularly those with apparently sporadic onset, in whom recessive AARS2 mutations should be suspected.

\section{Methods}

Patients were selected if there was clinical and radiologic evidence of ALSP, defined as an adult-onset disorder with prominent cognitive, neuropsychiatric, parkinsonian, or upper motor neuron signs accompanied by symmetric T2-weighted hyperintensity of the white matter on MRI with patchy areas of restricted diffusion. All patients had previously been shown

\section{Key Points}

Question What is the genetic cause of adult-onset leukoencephalopathy with axonal spheroids and pigmented glia (ALSP) in patients with typical features who are negative for mutations in the CSFIR (colony-stimulating factor 1) gene?

Findings In this case series study, using focused exome sequencing, bilallelic mutations in the AARS2 (alanyl-transfer [t]RNA synthetase 2) gene were identified in 5 patients with typical clinical, radiologic, and pathologic features of ALSP. Frameshifting and splice site mutations were common.

Meaning Screening the AARS2 gene should be considered in all patients with adult-onset leukodystrophy with clinical or radiologic features suggesting ALSP.

not to carry CSF1R mutations by results of conventional Sanger sequencing. This study was carried out with approval of the University College London Hospital Trust, and all patients gave written informed consent to participate. There was no financial compensation. Data were collected between May 2014 and September 2015 and analyzed between September 2015 and February 2016.

Focused exome sequencing was performed (Agilent Sure Select Focused Exome kit; Agilent Technologies) according to the manufacturer's protocol. After library preparation and capture, the pooled libraries were sequenced (Illumina HiSeq 1500; Illumina). FastQ sequencing data were aligned (Novoalign Software; Novocroft Technologies). Variants were extracted using the Mac model in SAMtools ${ }^{10}$ and calls were annotated using Annovar software. ${ }^{11}$ Mean target coverage was $40 \times$, with $92 \%$ of target bases reading more than $10 \times$.

If familial DNA was not available to phase compound heterozygous mutations, we cloned long polymerase chain reaction (PCR) products containing the genomic region spanning both variants into TOPO vectors (pCR2.1-TOPO TA vector; ThermoFisher Scientific), transformed bacteria, and used colony PCR to determine whether the variants were on the same or different alleles. We determined the impact of splice site variants by sequencing complementary DNA (cDNA) derived from patient tissue when available. Skin biopsies were performed and fibroblasts were cultured in a medium (Dulbecco modified Eagle medium; ThermoFisher Scientific) containing L-glutamine supplemented with $10 \%$ fetal calf serum. We then extracted RNA and reverse transcribed it to cDNA before PCR amplification and sequencing with cDNA-specific primers that spanned exon-exon junctions.

\section{Results}

\section{Clinical Descriptions}

Patient 1

This woman had normal childhood and adolescent development until her early 20s, when she was diagnosed with dermatomyositis and required treatment with corticosteroids, azathioprine sodium, and mycophenolate mofetil. She developed 


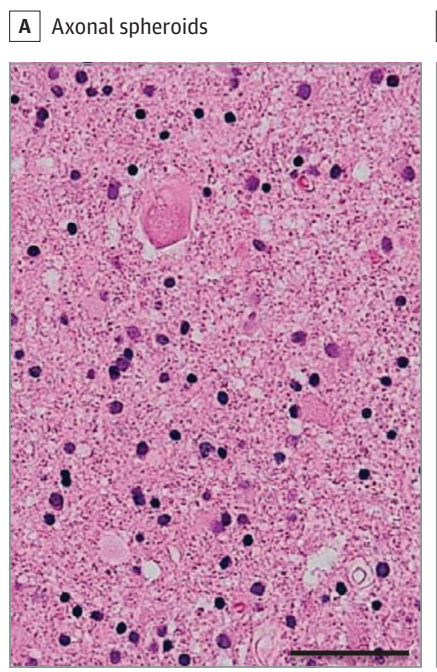

\section{B Pigmented cells}

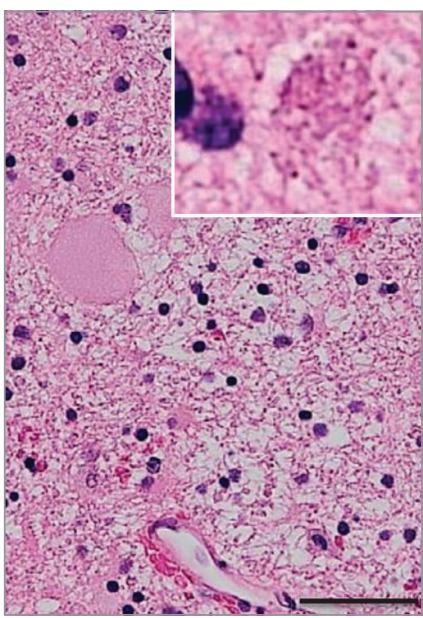

C Myelin pallor

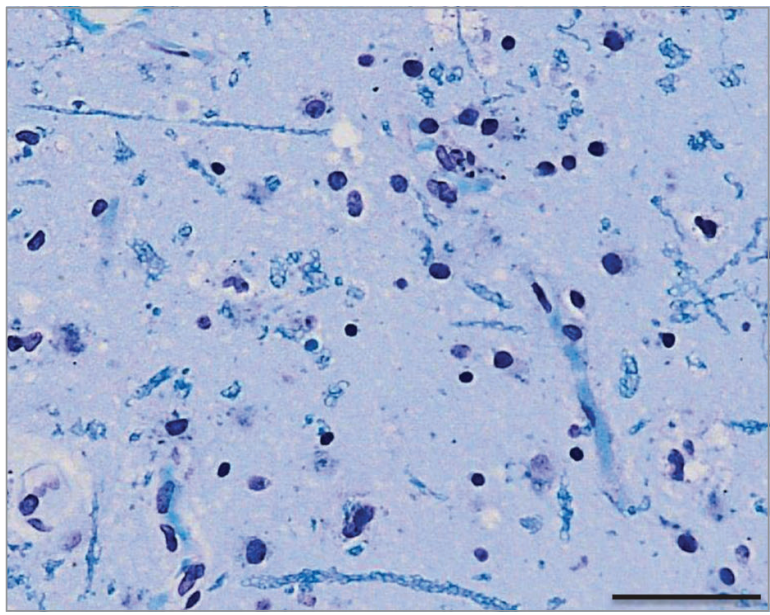

D Abnormal axonal profiles

E PAS positivity

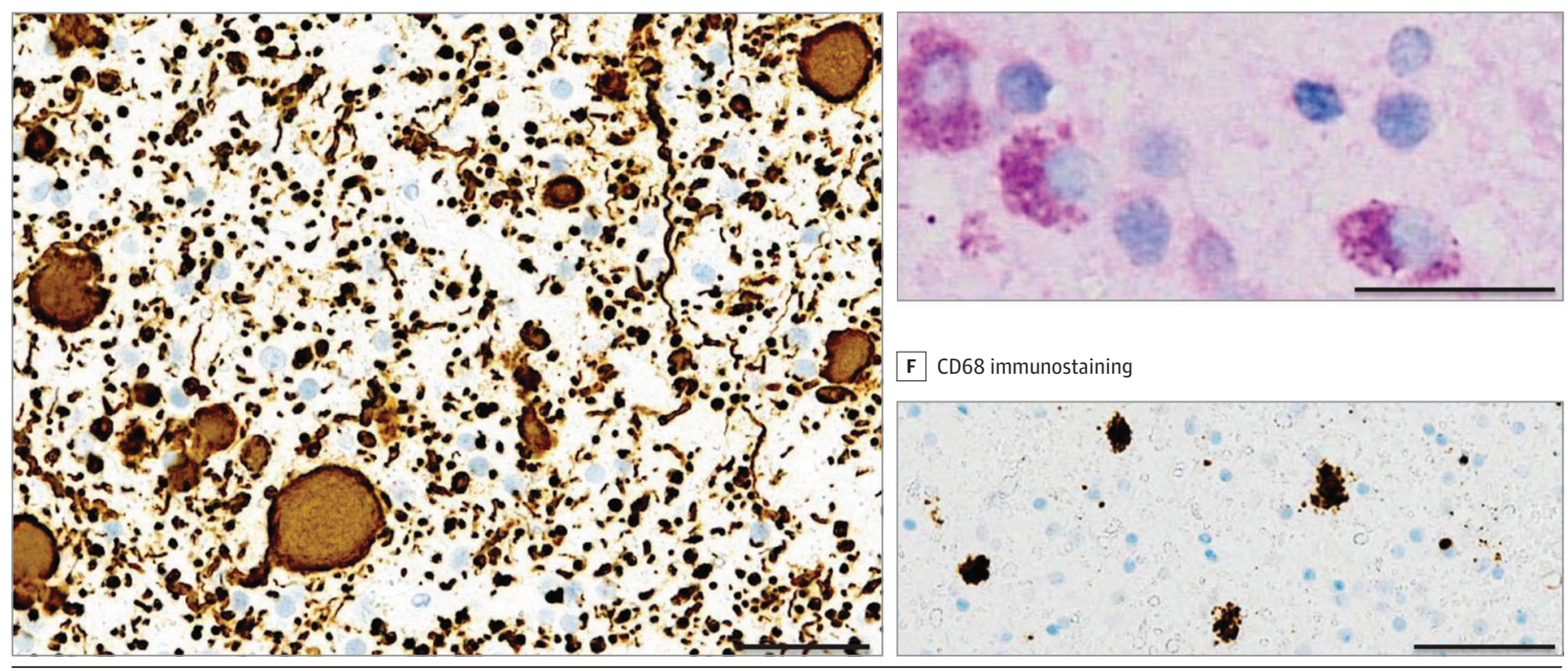

A and B, Rarified and gliotic white matter containing scattered eosinophilic axonal swellings (spheroids) and pigmented cells containing light brown lipopigment (B, inset). C, Myelin pallor and severe loss of myelinated axons. Immunostaining with an antibody against a cocktail of high- and low-molecular-weight neurofilaments showed numerous axonal spheroids with accentuated labeling at the periphery of the swellings. D, In addition, several

dystrophic, swollen, and beaded axonal profiles were seen. E, The pigmented cells were strongly positive for periodic acid-Schiff (PAS) staining and colocalized granular CD68 immunoreactivity (F), suggesting lysosomal localization. (A-D and F: hematoxylin-eosin, original magnification $\times 50$; E: Luxol fast blue, original magnification $\times 20$ ). premature ovarian failure in her mid-20s. Mild neuropsychiatric symptoms developed when she was in her early 40 s consisting of problems with mental calculation and generalized anxiety. These symptoms progressed and she became ataxic, with an asymmetric spastic paraparesis and left upper limb apraxia. Within 2 years of onset, there was marked apraxia in the left hand with weakness of finger extension and severe spasticity of the left leg. There were signs of a cortical sensory syndrome involving the parietal lobe with sensory inattention to the left. Magnetic resonance imaging scanning demonstrated symmetric confluent areas of high signal in the white matter on T2-weighted images. The presence of white matter abnormalities in any patient receiving long-term immunosuppression raised a concern for progressive multifocal leukoencephalopathy, which prompted a right parietal biopsy.

Right parietal biopsy findings demonstrated myelin loss with rarefaction and gliosis in the white matter and numerous axonal spheroids (Figure 1). The spheroids were accompanied by dystrophic axonal profiles and accumulated neurofilaments, p62, amyloid precursor protein, and $\beta$-amyloid. Scattered macrophages and microglia were also present and were intensely reactive for CD68. The macrophages accumulated light brown cytoplasmic lipopigment that was stained by periodic acid-Schiff, was diastase resistant, and was 
A T2 hyperintense white matter

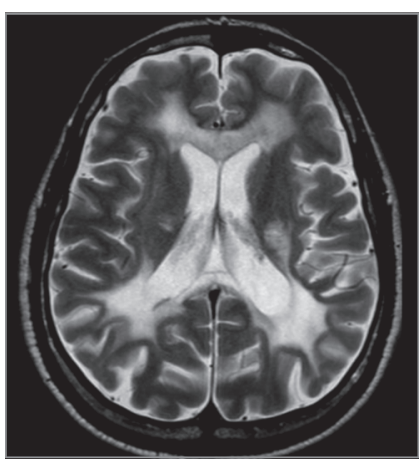

B FLAIR suppression

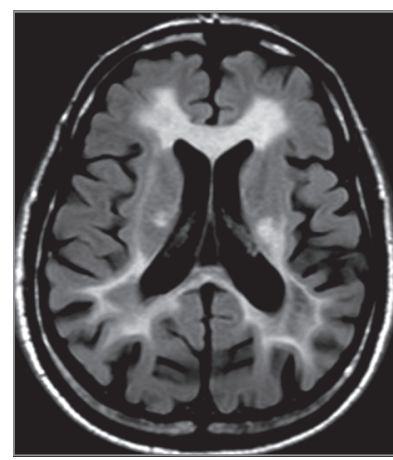

E Pyramidal tract involvement

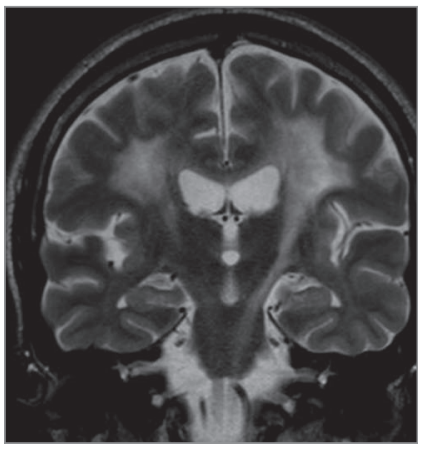

F Corpus callosum

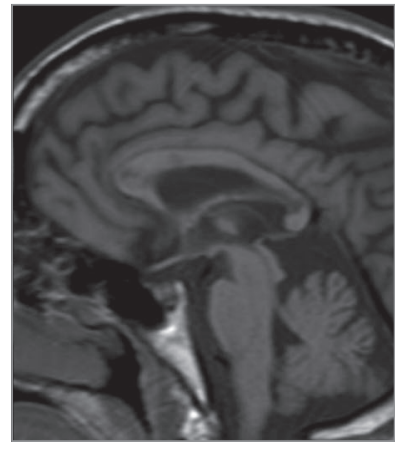

Asymmetric T2 hyperintensity in the deep and periventricular white matter (A) with fluid-attenuated inversion recovery (FLAIR) suppression (B) are noted in patient 2 , indicating white matter rarefaction. There are punctate areas of restricted diffusion seen in the diffusion-weighted imaging (DWI) (C) and apparent diffusion coefficient images at the level of the centrum semiovale (D).
C DWI restricted diffusion

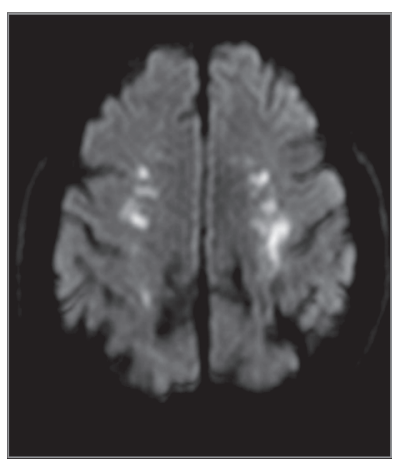

G DWI restricted diffusion

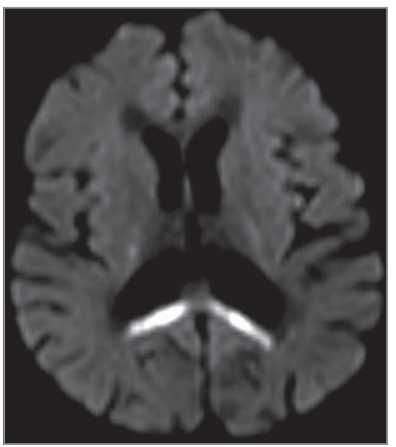

D Apparent diffusion coefficient

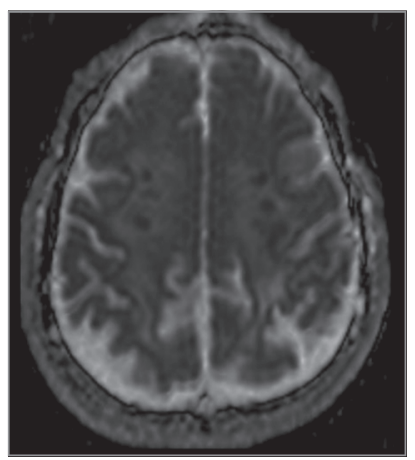

H Apparent diffusion coefficient

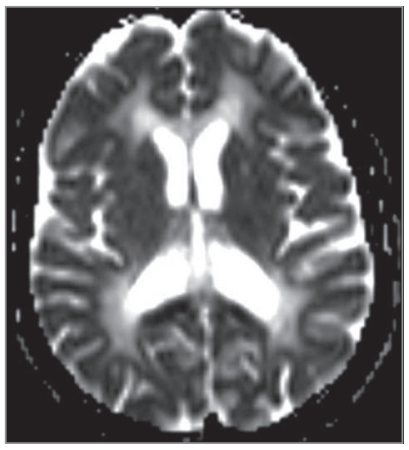

Asymmetric pyramidal tract involvement (E) with thinning of the corpus callosum and hypointense signal in the genu, body, and splenium (F). In patient 4, there was evidence of restricted diffusion in the splenium of the corpus callosum on the DWI (G) and apparent diffusion coefficient $(\mathrm{H})$ images.

intensely autofluorescent in unstained sections. Skeletal muscle biopsies taken at ages 20 and 40 years showed typical features of dermatomyositis as well as several fibers with complete or partial cyclooxygenase depletion (sequential cyclooxygenase-succinate dehydrogenase histochemistry). This defect was accentuated in the zones of perifascicular atrophy.

\section{Patient 2 and Patient 3}

Patient 2 was a man in his late 30s from Turkey who presented with a rapidly evolving neurologic syndrome with severe neuropsychiatric and behavioral changes. He developed obsessive behavior and hyperphagia with profound executive dysfunction and memory impairment. The behavioral changes required treatment with haloperidol. Examination revealed grossly normal tone and power, with a normal gait. There was an asymmetric, coarse upper limb tremor during rest, posture, and action. The syndrome rapidly progressed and he required residential care within 1 year of onset.

This patient's brother (patient 3) was similarly affected by a rapidly progressive neurodegenerative syndrome with onset in his mid-20s. We did not examine him. The family reported a similar disease, characterized by progressive diffi- culty with speech and swallowing with rapid motor decline. He became bed bound and died within 1 year of onset of the disease. Patient 3 did not experience the profound neuropsychiatric disorder found in his brother.

\section{Patient 4}

This patient was a young man of South Asian ancestry who had normal physical and cognitive development until middle adolescence, when he began to develop subtle psychiatric symptoms and decline in school performance. During the next 2 years, he became disinhibited and developed an ataxic gait with visual disturbance. Psychotic symptoms developed and he required treatment with quetiapine fumarate. There was no significant medical history and no signs of endocrine disease or hypogonadism. There was no family history of neurologic disease or known consanguinity. The examination revealed an asymmetric spastic paraparesis with bradykinesia and dystonic posturing of the hands. Cerebellar ataxia and dysdiadochokinesis were present with generalized hyperreflexia and bilateral extensor plantar responses. Moderate progression of symptoms occurred during the next 2 years, and the patient died in late adolescence from complications of the disease. 
Figure 3. Reverse-Transcriptase Polymerase Chain Reaction (RT-PCR) Analysis of Splice Site Variants

A c.1041-1G >A splicing effect

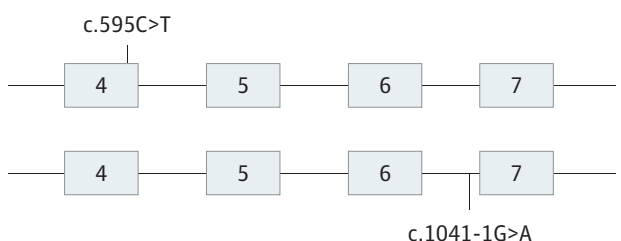

c. $1041-1 G>A$

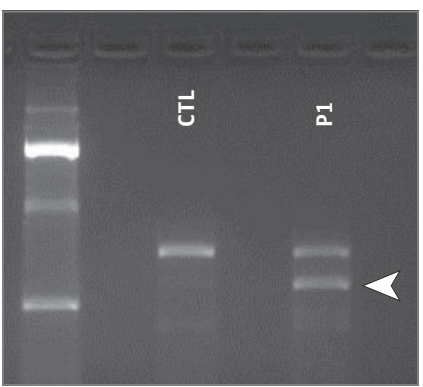

C G C C A I A A C A

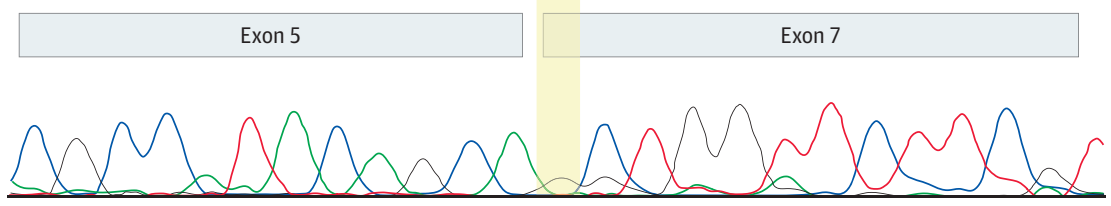

B c.1188G $>$ A splicing effect
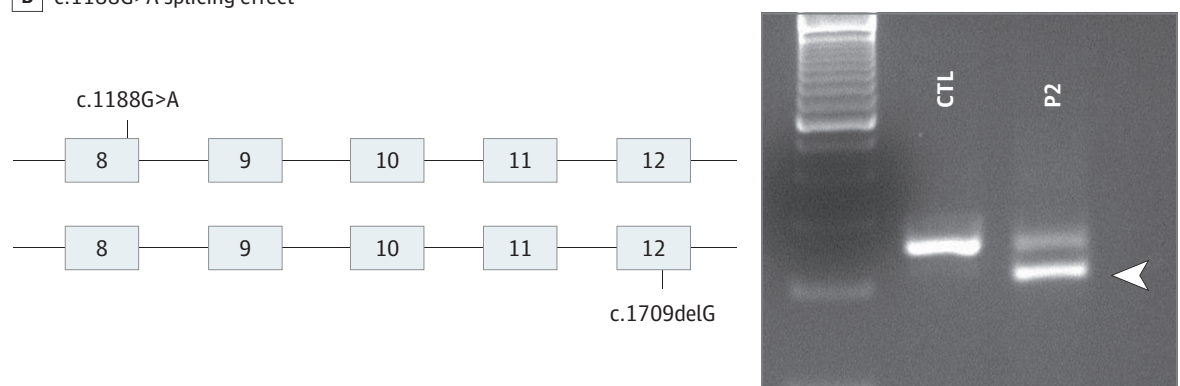

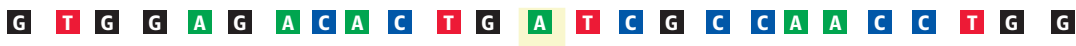

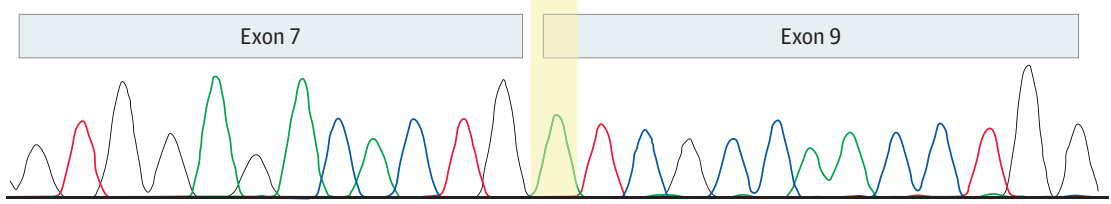

A, Schematic representation of the alanyl-transfer RNA synthetase 2 (AARS2) gene and results of RT-PCR analysis of complementary DNA (cDNA) from patient 1 (P1).

Polymerase chain reaction of a CDNA fragment spanning exons 3 to the junction of exons 9 and 10 produced 2 differently sized PCR products in $\mathrm{P} 1$ compared with the control (CTL). The lower band, indicated by the white arrowhead, was gel extracted and sequenced. Sequence analysis demonstrated that the lower band resulted from abnormal splicing with exclusion of exon 6. B, Results of PCR analysis of CDNA from patient 2 (P2). Reverse-transcriptase PCR of a CDNA fragment spanning exons 6 to the junction of exons 9 and 10 produced 2 differently sized $\mathrm{PCR}$ products in $\mathrm{P} 2$ compared with the CTL. The lower band, indicated by the white arrowhead, was gel extracted and sequenced. Sequence analysis demonstrated that the lower band resulted from abnormal splicing with exclusion of exon 8.

\section{Patient 5}

A man in his mid-40s from Turkey presented with a 1-year history of right upper limb dystonia and dysarthria. This development was followed by cognitive deterioration and neuropsychiatric symptoms including depression and aggressive behavior. He developed psychosis and was treated with olanzapine. There was no family history of neurologic disease and no known consanguinity. He had no significant medical history; in particular, there was no history of endocrine disease or hypogonadism. On examination, saccades were slowed and he had a pseudobulbar palsy. He exhibited an asymmetric spastic quadriparesis with prominent right-sided dystonia and parkinsonism. There was no weakness or sensory changes on examination. This patient's condition rapidly deteriorated and he died 1 year after symptom onset.

\section{MRI Findings}

Magnetic resonance imaging findings were consistent among all patients (Figure 2). The typical appearance was of asymmet- ric, confluent T2-weighted hyperintensity in the frontoparietal white matter with involvement of the immediate periventricular white matter in the most severely affected areas. Clear demonstration of white matter rarefaction in the peritrigonal regions that suppressed on fluid-attenuated inversion recovery sequences was seen in all cases. The subcortical U fibers were affected only in the areas of greatest signal abnormality. The corpus callosum was slightly thinned, with high signal in the genu, body, and splenium. Another consistent imaging feature was the asymmetric involvement of the pyramidal tracts extending into the posterior limb of the internal capsules and the brainstem. In all patients, there were punctate and partly confluent areas of restricted diffusion, seen especially in the centrum semiovale, corona radiate, and splenium of the corpus callosum. In patient 1 , for whom serial imaging was available, the areas of restricted diffusion persisted on an MRI for at least 7 months. None of the lesions enhanced after administration of intravenous gadolinium contrast. 
Table. Summary of Clinical, Radiologic, and Pathologic Findings in ALSP Due to CSF1R and AARS2 Mutations

\begin{tabular}{|c|c|c|}
\hline Feature & CSF1R & AARS2 \\
\hline $\begin{array}{l}\text { Mean age at } \\
\text { onset, } \\
\text { (range), y }\end{array}$ & $45(18-79)$ & $29(15-44)$ \\
\hline $\begin{array}{l}\text { Predominant } \\
\text { symptoms }\end{array}$ & $\begin{array}{l}\text { Psychiatric changes, } \\
\text { cognitive decline, } \\
\text { parkinsonism, pyramidal } \\
\text { signs, ataxia, and seizures }\end{array}$ & $\begin{array}{l}\text { Psychiatric changes, cognitive } \\
\text { decline, parkinsonism, pyramidal } \\
\text { signs, ataxia, and seizures; } \\
\text { ovarian failure is a distinguishing } \\
\text { feature in all women }\end{array}$ \\
\hline $\begin{array}{l}\text { Inheritance } \\
\text { pattern }\end{array}$ & $\begin{array}{l}\text { Autosomal dominant but } \\
\text { may appear sporadic }\end{array}$ & $\begin{array}{l}\text { Autosomal recessive but may } \\
\text { appear sporadic }\end{array}$ \\
\hline $\begin{array}{l}\text { Radiologic } \\
\text { appearance }\end{array}$ & $\begin{array}{l}\text { Largely symmetric } \\
\text { confluent T2 hyperintense } \\
\text { and T1 hypointense } \\
\text { WM signal change: } \\
\text { frontoparietal, corpus } \\
\text { callosum, and pyramidal } \\
\text { tracts; punctate to } \\
\text { confluent areas of } \\
\text { restricted diffusion on } \\
\text { DWI; and periventricular } \\
\text { calcifications reported in } \\
\text { some patients }\end{array}$ & $\begin{array}{l}\text { Largely symmetric confluent T2 } \\
\text { hyperintense/T1 hypointense } \\
\text { WM signal change: } \\
\text { frontoparietal, corpus callosum, } \\
\text { and pyramidal tracts; punctate } \\
\text { to confluent areas of restricted } \\
\text { diffusion on DWI; periventricular } \\
\text { FLAIR suppression due to WM } \\
\text { rarefaction is a distinguishing } \\
\text { feature }\end{array}$ \\
\hline $\begin{array}{l}\text { CNS } \\
\text { pathologic } \\
\text { features }\end{array}$ & $\begin{array}{l}\text { Axon and myelin loss; } \\
\text { frequent axonal spheroids } \\
\text { containing NF, p62, APP, } \\
\text { and } \beta \text {-amyloid; and } \\
\text { pigmented CD68-positive } \\
\text { microglia }\end{array}$ & $\begin{array}{l}\text { Axon and myelin loss; frequent } \\
\text { axonal spheroids containing NF, } \\
\text { p62, APP, and } \beta \text {-amyloid; } \\
\text { pigmented CD68-positive } \\
\text { microglia }\end{array}$ \\
\hline $\begin{array}{l}\text { Muscle } \\
\text { pathologic } \\
\text { features }\end{array}$ & None reported to date & $\begin{array}{l}\text { Diffusely reduced cytochrome-c } \\
\text { oxidase staining is a } \\
\text { distinguishing feature }\end{array}$ \\
\hline
\end{tabular}

Abbreviations: AARS2, alanyl-transfer RNA synthetase 2; ALSP, adult-onset leukoencephalopathy with axonal spheroids and pigmented glia; APP, amyloid precursor protein; CNS, central nervous system; COX, cyclooxygenase; CSF1R, colony-stimulating factor 1 receptor gene; DWI, diffusion-weighted imaging; FLAIR, fluid-attenuated inversion recovery; NF, neurofilaments; WM, white matter

\section{Genetic Findings}

We identified biallelic mutations in AARS2 in 5 patients from 4 families using focused exome sequencing. In patient 1 , we detected a novel c.1041-1G>A mutation in the splice acceptor site of exon 7. Analysis of cDNA from patient tissue confirmed that the mutation leads to abnormal splicing with exclusion of exon 6 (Figure 3). In trans was the previously reported c.595C > T, p. R199C variant.

In patients 2 and 3 , we detected a novel synonymous mutation affecting the last nucleotide of exon 8 (c.1188G $>$ A). Analysis of cDNA from fibroblasts of patient 2 demonstrated that the mutation leads to abnormal splicing with exclusion of exon 8 (Figure 3). In trans was another variant (c.1709delG) that leads to a frameshift with premature termination (p. G570Afs*21).

Patient 4 was found to carry the compound heterozygous c.[892_894del]; [2234_2235del] mutations. The c.892_894del leads to an inframe deletion of the last codon of exon $5 \mathrm{p}$. (298_298delQ) and may affect normal splicing of the transcript. The c.2234_2235 deletion leads to a frameshift with a predicted premature termination codon p.(Ser745Cysfs*60).
Tissue was not available to assess the variants' effect on RNA. Patient 5 was found to be homozygous for the c.595C>T, p.R199C variant, which was shown ${ }^{12}$ previously to affect the aminoacylation activity of the AARS2 protein.

\section{Discussion}

Adult-onset leukodystrophies and genetic leukoencephalopathies often present a diagnostic challenge with overlapping clinical phenotypes and MRI findings that lead to an expensive and lengthy diagnostic odyssey. ${ }^{13}$ The identification and characterization of the ALSP-associated CSF1R gene provided many patients and families with a precise genetic diagnosis, although many adult-onset leukodystrophies remain undefined. The use of exome sequencing has advanced our diagnostic capabilities with the discovery of several new leukodystrophy genes, and deep clinical and radiologic phenotyping are leading to improved diagnostic rates. ${ }^{14,15}$ In this study, we show that typical ALSP cases can be caused by mutations in CSF1R and AARS2, with MRI findings that typically demonstrated an asymmetric confluent T2 hyperintensity in the frontoparietal and periventricular white matter with rarefaction.

Neuroaxonal swellings, the larger of which are known as spheroids, are a morphologic abnormality of axons of uncertain pathogenesis with proposed wallerian and nonwallerian mechanisms ${ }^{9}$ that can occur in the central and peripheral nervous systems. Spheroids are associated with a variety of conditions, including physiologic aging, acute axonal injury, as being around infarcts or foci of demyelination, and as an integral feature in certain heritable neurodegenerative conditions predominantly affecting the gray matter as with neurodegeneration with brain iron accumulation ${ }^{16,17}$ or the white matter as in leukodystrophy. The combination of adult-onset leukodystrophy with axonal spheroids and pigmented microglia has been believed to be highly suggestive, if not pathognomonic, of CSF1R-related disease..$^{3,18-20}$ In this study, we show that these pathologic changes are not specific and that mutations in AARS2 can lead to an identical clinical, radiologic, and tissue phenotype to ALSP. In women, at least, the presence of premature ovarian failure may provide a clue that $A A R S 2$ is responsible, but no such clue exists for men (Table).

\section{Conclusions}

We advocate for increasing adoption of next-generation sequencing approaches in the routine care of patients with genetic leukoencephalopathies. However, when such approaches are not available, we recommend screening for mutations in CSF1R and AARS2 in patients believed clinically or pathologically to have ALSP.
ARTICLE INFORMATION

Accepted for Publication: May 12, 2016.
Published Online: October 17, 2016. doi:10.1001/jamaneurol.2016.2229

Author Affiliations: Department of Molecular Neuroscience, University College London Institute of Neurology, London, England (Lynch, Zhang, Houlden); The Leonard Wolfson Experimental Neurology Centre, University College London Institute of Neurology, London, England (Lynch); 
Department of Neurology, Royal Free Hospital, London, England (Zhang, Bhattacharjee, Wilson); Lysholm Department of Neuroradiology, The National Hospital for Neurology and Neurosurgery, London, England (Lakshmanan, Adams, Davagnanam); Dementia Research Centre, Department of Neurodegeneration, The National Hospital for Neurology and Neurosurgery. University College London Institute of Neurology, London, England (Kinsella, Fox); University College Dublin, Department of Neurology, St Vincent's University Hospital, Dublin, Ireland (Kinsella); Department of Neurology, Istanbul Faculty of Medicine, Istanbul University, Istanbul, Turkey (Uzun, Karbay, Tüfekçioğlu, Hanağası); Department of Neurology, Queen Alexandra Hospital, Portsmouth, England (Burke, Hammans); Wessex Neurological Centre, University Hospital Southampton, Southampton, England (Burke); Wessex Clinical Genetics Services, Princess Anne Hospital, Academic Unit of Human Development and Health, Faculty of Medicine, University of Southampton, Southampton, England (Foulds); Clinical Neurosciences, Clinical \& Experimental Sciences, University of Southampton, Department of Cellular Pathology, Southampton General Hospital, Southampton, England (Walker, Nicoll); Department of Neuroinflammation, University College London Institute of Neurology, London, England (Chataway); Division of Neuropathology and Department of Neurodegenerative Disease, The National Hospital for Neurology and Neurosurgery, University College London Institute of Neurology, London, England (Phadke); Neurogenetics Laboratory, The National Hospital for Neurology and Neurosurgery, London, England (Houlden)

Author Contributions: Drs Lynch and Houlden had full access to all the data in the study and take responsibility for the integrity of the data and the accuracy of the data analysis.

Study concept and design: Lynch, Houlden.

Acquisition, analysis, or interpretation of data: All authors.

Drafting of the manuscript: Lynch, Lakshmanan, Chataway, Fox, Davagnanam, Phadke, Houlden. Critical revision of the manuscript for important intellectual content: Lynch, Zhang, Lakshmanan, Kinsella, Uzun, Karbay, Tüfekçioğlu, Hanağası,

Burke, Foulds, Hammans, Bhattacharjee, Wilson, Adams, Walker, Nicoll, Fox, Davagnanam. Statistical analysis: Lynch.

Obtained funding: Houlden.

Administrative, technical, or material support: Lynch, Uzun, Karbay, Tüfekçioğlu, Hanağası, Bhattacharjee, Walker, Nicoll, Fox, Phadke. Study supervision: Chataway, Fox, Davagnanam, Houlden.

Conflict of Interest Disclosures: Dr Chataway has received support from Novartis, Teva, and Sanofi.
Dr Fox has received support from Novartis, Sanofi, Genentech, and Janssen. No other disclosures were reported.

Funding/Support: This study was supported by the Medical Research Council, The Wellcome Trust and Synaptopathies strategic award 104033, The Brain Research Trust, The UK HSP Society, and the European Union FP7/2007-2013 under grant agreement number 2012-305121 from Neuromics. The study was also supported by the Leonard Wolfson Experimental Neurology Centre and the National Institute for Health Research University College London Hospitals Biomedical Research Centre.

Role of the Funder/Sponsor: The funding sources had no role in the design and conduct of the study: collection, management, analysis, and interpretation of the data; preparation, review, or approval of the manuscript; and decision to submit the manuscript for publication.

Additional Contributions: We thank the patients and their families for granting permission to publish this information.

\section{REFERENCES}

1. Vanderver A, Prust M, Tonduti D, et al; GLIA Consortium. Case definition and classification of leukodystrophies and leukoencephalopathies. Mol Genet Metab. 2015;114(4):494-500.

2. Ayrignac $X$, Carra-Dalliere C, Menjot de Champfleur $\mathrm{N}$, et al. Adult-onset genetic leukoencephalopathies: a MRI pattern-based approach in a comprehensive study of 154 patients. Brain. 2015;138(Pt 2):284-292.

3. Rademakers R, Baker M, Nicholson AM, et al. Mutations in the colony stimulating factor 1 receptor (CSF1R) gene cause hereditary diffuse leukoencephalopathy with spheroids. Nat Genet. 2011;44(2):200-205.

4. Sundal C, Fujioka S, Van Gerpen JA, et al. Parkinsonian features in hereditary diffuse leukoencephalopathy with spheroids (HDLS) and CSF1R mutations. Parkinsonism Relat Disord. 2013; 19(10):869-877.

5. Lynch DS, Jaunmuktane Z, Sheerin U-M, et al. Hereditary leukoencephalopathy with axonal spheroids: a spectrum of phenotypes from CNS vasculitis to parkinsonism in an adult onset leukodystrophy series. J Neurol Neurosurg Psychiatry. 2016;87(5):512-519.

6. Foulds N, Pengelly RJ, Hammans SR, et al. Adult-onset leukoencephalopathy with axonal spheroids and pigmented glia caused by a novel R782G mutation in CSF1R. Sci Rep. 2015;5:10042.

7. Fernández-Vega I, Pérez de Heredia-Goñi K, Santos-Juanes J, et al. Sporadic adult-onset leucodystrophy with axonal spheroids and pigmented glia with no mutations in the known targeted genes. Histopathology. 2016;68(2) 308-312.
8. Götz A, Tyynismaa H, Euro L, et al. Exome sequencing identifies mitochondrial alanyl-tRNA synthetase mutations in infantile mitochondrial cardiomyopathy. Am J Hum Genet. 2011;88(5): 635-642.

9. Dallabona C, Diodato D, Kevelam $\mathrm{SH}$, et al. Novel (ovario) leukodystrophy related to AARS2 mutations. Neurology. 2014;82(23):2063-2071.

10. Li H, Handsaker B, Wysoker A, et al; 1000 Genome Project Data Processing Subgroup. The Sequence Alignment/Map (SAM) format and SAMtools. Bioinformatics. 2009;25:2078-2079.

11. Wang K, Li M, Hakonarson H. ANNOVAR: functional annotation of genetic variants from high-throughput sequencing data. Nucleic Acids Res. 2010;38(16):e164.

12. Euro L, Konovalova S, Asin-Cayuela J, et al. Structural modeling of tissue-specific mitochondrial alanyl-tRNA synthetase (AARS2) defects predicts differential effects on aminoacylation. Front Genet. 2015;6(2):21.

13. Parikh S, Bernard G, Leventer RJ, et al; GLIA Consortium. A clinical approach to the diagnosis of patients with leukodystrophies and genetic leukoencephelopathies. Mol Genet Metab. 2015;114 (4):501-515.

14. Scheper GC, van der Klok T, van Andel RJ, et al Mitochondrial aspartyl-tRNA synthetase deficiency causes leukoencephalopathy with brain stem and spinal cord involvement and lactate elevation. Nat Genet. 2007;39(4):534-539.

15. Steenweg ME, Ghezzi D, Haack T, et al. Leukoencephalopathy with thalamus and brainstem involvement and high lactate 'LTBL' caused by EARS2 mutations. Brain. 2012;135(Pt 5): 1387-1394.

16. Ryu J, Horkayne-Szakaly I, Xu L, et al. The problem of axonal injury in the brains of veterans with histories of blast exposure. Acta Neuropathol Commun. 2014;2:153.

17. Nardocci N, Zorzi G, Farina L, et al. Infantile neuroaxonal dystrophy: clinical spectrum and diagnostic criteria. Neurology. 1999;52(7):1472-1478.

18. Guerreiro R, Kara E, Le Ber I, et al. Genetic analysis of inherited leukodystrophies: genotype-phenotype correlations in the CSF1R gene. JAMA Neurol. 2013;70(7):875-882.

19. Freeman $S H$, Hyman $B T$, Sims KB, et al. Adult onset leukodystrophy with neuroaxonal spheroids: clinical, neuroimaging and neuropathologic observations. Brain Pathol. 2009;19(1):39-47.

20. Baba Y, Ghetti B, Baker MC, et al. Hereditary diffuse leukoencephalopathy with spheroids: clinical, pathologic and genetic studies of a new kindred. Acta Neuropathol. 2006;111(4):300-311. 